\title{
A low donor kidney weight to recipient weight ratio is associated with decreased long-term graft survival
}

Incompatibility between the weight of a donor kidney and the weight of the transplant recipient is an independent predictor of long-term renal allograft survival, say researchers in France.

In a study reported in 2005, Magali

Giral et al. found that a low donor kidney weight to recipient weight (Kw:Rw) ratio did not affect graft survival. As mean follow-up in that study was only 32 months, however, the researchers wanted to assess longer term consequences of a low $\mathrm{Kw}$ : Rw ratio.

In the present study, the investigators analyzed data from 1,060 renal transplant recipients who had received a kidney graft between April 1995 and January 2006.

Mean follow-up was 6.2 years.

Giral et al. found that in patients with a low $\mathrm{Kw}$ :Rw ratio $(<2.3 \mathrm{~g} / \mathrm{kg})$, estimated glomerular filtration rate (eGFR) increased by a mean of $5.74 \mathrm{ml} / \mathrm{min}$ between 3 months and 6 months after transplantation, reached a plateau at
6 months, and decreased rapidly after 7 years at a mean rate of $3.17 \mathrm{ml} / \mathrm{min}$ per year. Among recipients with a high $\mathrm{Kw}$ :Rw ratio $(\geq 2.3 \mathrm{~g} / \mathrm{kg})$, the mean adaptive increase in eGFR between 3 months and 6 months was much lower $(3.22 \mathrm{ml} / \mathrm{min})$ and eGFR decreased at a much slower rate after 7 years $(1.34 \mathrm{ml} / \mathrm{min}$ per year).

\section{4 ....avoiding kidney and} recipient weight incompatibility may improve late clinical outcome... 77

The researchers also found that patients with a low $\mathrm{Kw}$ :Rw ratio had an increased risk of proteinuria $>0.5 \mathrm{~g}$ per day at 1 year and were more likely than patients with a high $\mathrm{Kw}$ :Rw ratio to receive antihypertensive agents. Examination of 141 graft biopsy samples revealed that segmental and global glomerulosclerosis were more common in patients with a low Kw:Rw ratio. A Cox multivariate analysis showed that a Kw:Rw ratio $<2.3 \mathrm{~g} / \mathrm{kg}$ was independently associated with a $55 \%$ increase in the risk of transplant failure after 2 years. "...incompatibility between graft and recipient weight is an independent predictor of long-term graft survival, suggesting that avoiding kidney and recipient weight incompatibility may improve late clinical outcome after kidney transplantation," note the researchers. "It will be important to replicate these provocative observations ... in other large cohorts of patients with sufficiently long follow-up," states Ron Shapiro of the University of Pittsburgh in an accompanying editorial.

\section{Rebecca Ireland}

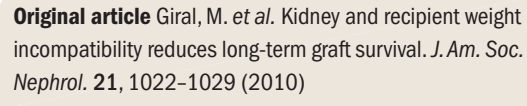

Original article Giral, M. et al. Kidney and recipient weight incompatibility reduces long-term graft survival. J. Am. Soc. Nephrol. 21, 1022-1029 (2010) 